\title{
Des cailles et de la ciguë. A propos de l'Anonyme de Londres (P.Lond.Lit. 165, Brit.Libr. inv. 137) col. XXVI，16-19
}

\author{
Véronique Boudon-Millot
}

Découvert il y a plus d'un siècle, l'Anonyme de Londres (P.Lond.Lit. 165, Brit.Libr. inv. 137), le plus célèbre des papyrus sur la médecine ancienne qui nous soit parvenu, vient de faire l'objet de deux éditions critiques récentes, publiées respectivement par D. Manetti en 2011 et par A. Ricciardetto en $2016^{1}$. Cette dernière édition en particulier, parce qu'elle est accompagnée de la première traduction française, offre désormais au lecteur tous les moyens non seulement de découvrir mais aussi de faire progresser la connaissance d'un texte majeur pour l'histoire de la médecine et de la pensée médicale. Longtemps considéré comme "une copie, faite par un étudiant en médecine, de notes de cours prises par un autre étudiant», ce texte est aujourd'hui plus volontiers abordé, notamment à la suite des recherches de D. Manetti ${ }^{2}$, comme une œuvre originale formant un tout composé de trois parties distinctes (nosologie, étiologie et physiologie $)^{3}$. La présente contribution voudrait attirer l'attention sur un des exemples cités par l'auteur de l'Anonyme à propos de l'absurdité de la théorie médicale du médecin Asclépiade de Bithynie, dans le but de mettre en évidence l'originalité de l'auteur de l'Anonyme.

Le passage qui nous intéresse (col. xxvi, 16-19) se situe à l'intérieur de la troisième section traditionnellement qualifiée de «physiologique» dont A. Ricciardetto note avec justesse (p. XCIX) qu' «elle a été très peu étudiée, bien qu'elle représente une source précieuse pour la connaisssance des médecins du $\mathrm{IV}^{\mathrm{e}}$ siècle avant J.-C. et de la période hellénistique, dont les œuvres, à

\footnotetext{
${ }^{1}$ Edité pour la première fois par Hermann Diels en 1893 dans le Supplementum Aristotelicum III, 1 (texte grec sans traduction), l'Anonyme de Londres a fait l'objet d'une nouvelle édition critique par Manetti, Anonymus (2011, texte grec seul), puis par Ricciardetto, Anonyme (2016, texte grec et traduction française), cette dernière ayant été précédée en juin 2014 d'une édition grand format publiée par le même auteur dans la collection «Papyrologica Leodiensia » des Presses Universitaires de Liège.

${ }^{2}$ Manetti, Autografi.

${ }^{3}$ Toutefois, et comme le note également Marganne, Acquisition, 167, la nature exacte du texte reste discutée : pour Manetti, Anonymus, le rouleau pourrait contenir « le brouillon d'un ouvrage »; pour Ricciardetto, Anonyme, il s'agirait d'« un exercice à la dialectique pour soi-même, peut-être en vue de la préparation d'un concours ».
} 
l'exception de celles d'Aristote, sont presque entièrement perdues ». Dans cette section, l'auteur de l'Anonyme ne se contente plus de présenter les théories de ses prédécesseurs (doxographie), il raisonne et argumente pour son propre compte, et en particulier il ne se prive pas de polémiquer avec Asclépiade, dont il entreprend de réfuter une partie des théories sur la distribution et l'assimilation des aliments à travers le corps. D. Manetti a déjà attiré l'attention sur ce passage dont elle a proposé une analyse très éclairante ${ }^{4}$. L'auteur de l'Anonyme va en effet s'efforcer de réfuter la théorie mécanique d'Asclépiade selon laquelle il n'existe pas de coction (ou de transformation qualitative) des aliments lors de la digestion qui, dès lors, se réduit à un simple processus de division et de liquéfaction de la nourriture. Une des conséquences de cette théorie, qui prend à contre-pied le principe hippocratico-galénique de la transformation des aliments au cours de la coction, est qu'il ne saurait y avoir de réelle différence qualitative entre les matières ingérées et expulsées et que, par conséquent, les excréments sont aussi potentiellement de la nourriture ${ }^{5}$.

L'auteur de l'Anonyme entreprend alors de discuter la théorie d'Asclépiade avant de recourir à un raisonnement par l'absurde. Il commence d'abord par tirer toutes les conséquences de cette théorie : certains animaux privés de raison se nourrissent d'excréments ${ }^{6}$, on peut donc en déduire que les excréments ( $\tau \grave{\alpha}$

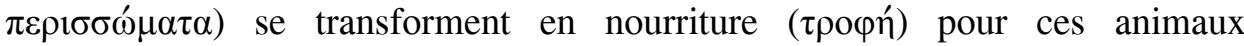
coprophages, nourriture qui elle-même se transforme en chair ( бó $\rho \kappa \alpha)$, avant d'être consommée par l'homme pour produire sa propre croissance. Et «par le même raisonnement », on affirmera aussi que « les résidus des hommes sont, diton, une nourriture $»^{7}$. Cependant, dès lors que l'on cherche à tirer de ces observations un principe universel, on aboutit nécessairement à un non-sens. L'auteur de l'Anonyme choisit alors de citer deux exemples :

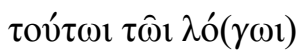

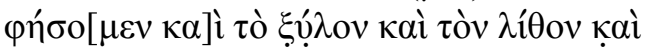

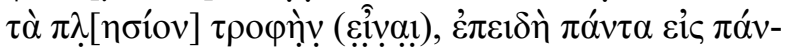

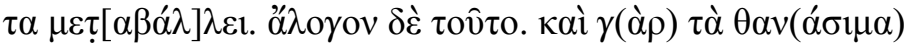

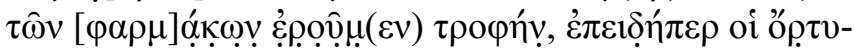

\footnotetext{
${ }^{4}$ Manetti, Asclepiade, qui toutefois observe (p. 340) que le lien entretenu entre ce développement et celui qui précède n'est pas parfaitement clair.

${ }^{5}$ Cette théorie d'Asclépiade est notamment confirmée par Caelius Aurelianus, Acut. I 114 (CML VI 1, p. 86 Bendz) : praeterea excrementa ventris, Graeci scybala dicunt, negat aliena esse natura, siquidem etiam ex ipsis corpora augeantur. quaedam denique, inquit, animalia ex ipsis solummodo nutriunt $\langle$ ur $\rangle$.

${ }^{6}$ Même si l'auteur de l'Anonyme ne donne ici aucun exemple, la coprophagie est effectivement un mode d'alimentation normal observé, outre chez certains insectes, à la fois chez les carnivores (porcs) et les oiseaux.

${ }^{7}$ Anon.Lond., xxvi 8-9 (traduction personnelle légèrement modifiée).
} 


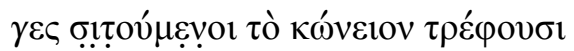

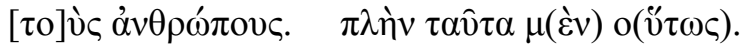

Par ce raisonnement, nous dirons aussi que le bois, la pierre et leurs semblables sont de la nourriture, puisque tout se transforme en tout. Mais cela est absurde. Et de fait, nous dirons que, parmi les drogues, les mortelles sont une nourriture puisque les cailles qui mangent la ciguë nourrissent les hommes. Excepté cela, il en va ainsi ${ }^{8}$.

Le parallélisme entre ces deux exemples est souligné par l'emploi du verbe

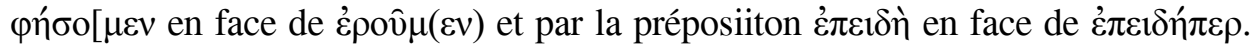
L'auteur de l'Anonyme énonce donc deux exceptions susceptibles de ruiner un tel raisonnement et recourt à deux exemples empruntés l'un au non vivant (matière inanimée), l'autre au vivant (matière animale ou végétale). Ces deux exceptions sont en outre de nature différente, puisque la première est relative à la substance (bois, pierre) et la seconde à la qualité (comestible ou non). De fait, tout ne peut pas se transformer en nourriture, soit parce que la matière ingérée n'est pas transformable (bois, pierre), soit parce qu'elle n'est pas comestible par celui qui la consomme. Qu'en est-il cependant du sens exact de ce dernier exemple et de cette chair des cailles mangeuses de ciguë dont se repaissent les hommes?

\section{De l'hellébore pour les cailles et de la ciguë pour les étourneaux.}

Dès l'antiquité, l'exemple de l'animal qui se nourrit de substances toxiques tout en restant consommable pour l'homme n'a cessé d'éveiller la curiosité des médecins. Le cas des oiseaux, en particulier, cailles ou étourneaux, qui se nourrissent de graines de jusquiame, de ciguë ou d'hellébore, tout en restant comestibles pour l'homme, a bien été observé et décrit par les médecins". Toutefois, la mention de la ciguë à propos des cailles ne manque pas de surprendre $^{10}$. En effet, dans la littérature médicale, ce sont d'ordinaire les étourneaux ( $\psi \hat{\eta} \rho \varepsilon \varsigma)$ qui consomment la ciguë et non les cailles (ő $\rho \tau \gamma \varepsilon \varsigma)$ qui, elles, se nourrissent d'hellébore. La lecture du mot ö $\rho \tau v \gamma \varepsilon \varsigma$ dans ce passage, il est vrai fortement endommagé, du papyrus ne fait pourtant plus guère de doute

\footnotetext{
${ }^{8}$ Anon.Lond., xxvi 13-19 (traduction personnelle modifiée) : comparer pour ce dernier membre de phrase la traduction de Manetti, Asclepiade, 342 («Ma basta cosí su questo ») et de Ricciardetto, Anonyme, 36 ( «Au reste, il en est ainsi »). Je comprends pour ma part que $\tau \alpha \hat{\tau} \tau \alpha$ renvoie aux trois exceptions énoncées juste avant (bois, pierre et drogues mortelles qui ne peuvent jamais être de la nourriture pour les hommes).

${ }^{9}$ Sur ces deux espèces d'oiseaux, voir Thompson, Glossary, 123-6 (sur les cailles) et 198 (sur les étourneaux). Sur la jusquiame, voir André, Plantes, 127 ; sur la ciguë, ibid., 73 ; sur l'hellébore ibid., 94.

${ }^{10}$ Sur la ciguë et les troubles qu'elle entraîne, voir Nicandre, Alexiph. 186-206 (éd. J.-M. Jacques, Paris, CUF, 2007, pp. 18-20) et Aétius, XIII 65 (ibid., pp. 256-7).
} 
depuis la restitution de P. Kotsia-Pantele ${ }^{11}$.

La consommation d'hellébore par les cailles avait déjà été notée par l'auteur du traité pseudo-aristotélicien Sur les plantes I 5 (820b6-7) où la jusquiame est citée aux côtés de l'hellébore, à l'exclusion de la ciguë : " La jusquiame et l'hellébore sont nuisibles aux hommes, mais de la nourriture pour les cailles »

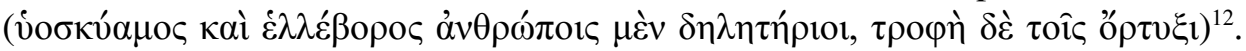
Le fait a d'ailleurs été encore relevé au $\mathrm{VI}^{\mathrm{e}}$ siècle par le philosophe et commentateur néoplatonicien Asclepius Trallianus ${ }^{13}$. Il est également rapporté par Lucrèce au $\mathrm{I}^{\mathrm{er}}$ siècle de notre ère ${ }^{14}$. Mais surtout, il est confirmé par Galien en plusieurs endroits dans des contextes comparables à celui de l'Anonyme.

Il s'agit en effet à chaque fois, pour le médecin de Pergame, d'expliquer comment certains êtres vivants peuvent être capables d'assimiler des substances qui, pour d'autres êtres, s'avèrent dangereusement toxiques et même parfois mortelles. Dans les Facultés des aliments, Galien attribue cette particularité à l'affinité entretenue entre certaines nourritures et certaines espèces et qui fait qu' ânes et chevaux préfèrent la paille, mais que le lion préfèrera la chair crue et l'homme la chair cuite et le pain issu des graines céréalières. «Et d'ailleurs, ajoute Galien, quand les cailles mangent l'hellébore et les étourneaux la ciguë, ils n'en souffrent pas, non plus que les bœufs quand ils mangent l'ers $»^{15}$. Galien, dans le Sur les tempéraments, explique cette affinité entre les aliments et les êtres par leur tempérament plus ou moins chaud, froid, sec ou humide. En particulier, si « la ciguë est une nourriture pour l'étourneau, mais un poison pour l'homme, et si pour les cailles l'hellébore est une nourriture, mais pour les hommes un poison, c'est que le tempérament des cailles est capable d'assimiler l'hellébore, alors que celui des hommes en est incapable $»^{16}$. De fait, explique Galien dans les Facultés des médicaments simples, «ce n'est pas parce que la

${ }^{11}$ Kotsia-Pantele, P.Lond. 137. H. Diels, le premier éditeur, déchiffrait ol̋otoc, «suint » ou « graisse » de la laine.

${ }^{12}$ Sur l'histoire compliquée du Traité sur les plantes, voir Drossaart / Poortman, De plantis (Textes en syriaque, arabe, latin, hébreu, grec, avec trad., notes et commentaires en anglais).

13 Voir Asclepii in Aristotelis metaphysicorum libros A-Z commentaria [Commentaria in

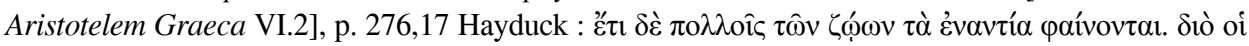

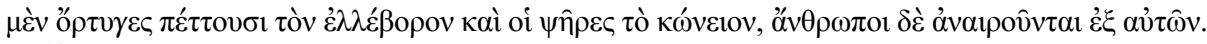

${ }^{14}$ Lucr. R.Nat. IV 641 : praeterea nobis veratrum est acre venenum, at capris adipes et cocturnicibus auget («De même, l'hellébore qui est pour nous un poison violent, engraisse néanmoins les chèvres et les cailles »).

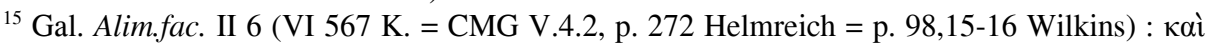

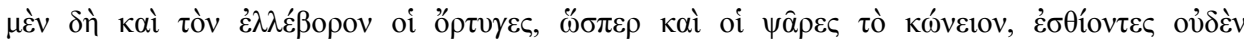

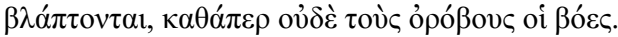

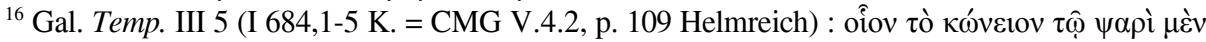

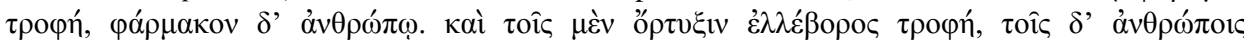

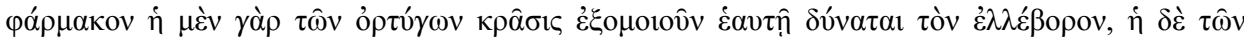

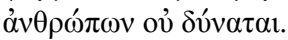


ciguë est refroidissante pour l'homme, qu'elle l'est aussi pour l'étourneau ; et ce n'est pas parce qu'elle est nourrissante pour l'étourneau qu'elle l'est aussi pour l'homme; pour les mêmes raisons, ce n'est pas parce que l'hellébore est purgative < pour les hommes> qu'elle l'est aussi pour les cailles, et ce n'est pas parce qu'elle est nourrissante pour ces dernières, qu'elle l'est aussi pour les hommes ${ }^{17} \gg$. Parfois, ces différences de tempérament sont observables non seulement entre espèces mais aussi entre individus d'une même espèce, comme Galien le précise un peu plus loin dans le même traité à propos d'un autre purgatif, le grain de Cnide (notre garou ou sainbois ${ }^{18}$ ) qui, chez les rares individus qui parviennent à en triompher, « au lieu de faire l'effet d'un purgatif, parfois se mue en nourriture, comme toujours l'hellébore l'est (sc. une nourriture) pour les cailles $»^{19}$.

Parfois enfin la faculté d'un aliment se transmet indirectement d'individu à individu, notamment par la voie de l'allaitement. Cette précision est donnée par Galien dans son commentaire au passage d'Epidémies VI où l'auteur hippocratique affirme que «Une femme, une chèvre ayant mangé de l'élatèrion ou du concombre sauvage, les enfants sont purgés $»^{20}$. Cet effet laxatif du lait de chèvre ou du lait maternel, dû à la consommation par la mère d'aliments euxmêmes utilisés pour leur propriété laxative, était cependant discuté. Certains, commente Galien, estiment qu'aucune des propriétés présentes au départ dans les aliments ne peut se conserver après coction ou sanguification, tandis que d'autres estiment que les facultés fortes demeurent pendant longtemps ${ }^{21}$. Et il remarque que chez lui (sc. en Asie) au printemps quand les chèvres broutent des jeunes pousses de scammonée ${ }^{22}$, leur lait devient laxatif ${ }^{23}$. Et en particulier à propos des cailles qui ont mangé de l'hellébore, il ajoute :

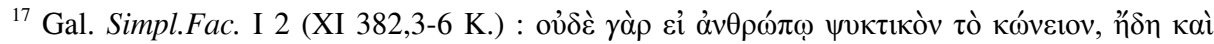

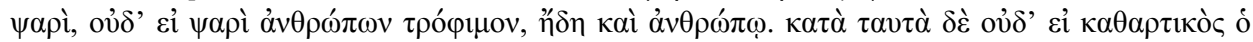

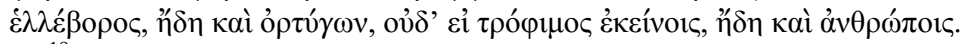

${ }^{18}$ Sur le grain de Cnide (Daphne gnidium L.), voir André, Plantes, 313 et Nissen, Asclépios, 19 : «La dénomination 'grain de Cnide' s'applique plus exactement aux fruits de cet arbrisseau, en l'occurrence des baies ovoïdes rouges hautement toxiques, à l'instar du reste de la plante, très vénéneuse. Les baies de garou ont pourtant été utilisées par les médecins tout au long de l'Antiquité pour leurs propriétés purgatives très appréciées dans la thérapeutique. »

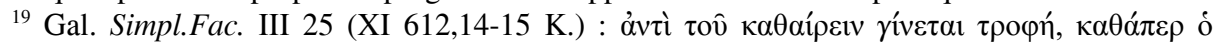

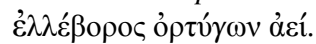

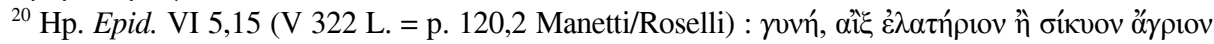

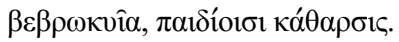

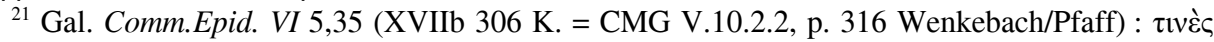

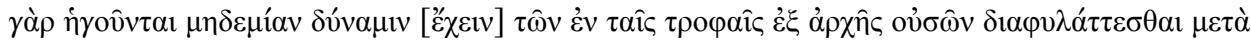

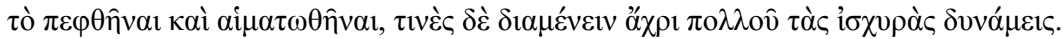

${ }^{22}$ Sur la scammonée, voir André, Plantes, 228. 
Et assurément en Doride, en Béotie, en Thessalie et dans toutes les régions qui en sont voisines, beaucoup furent pris de tensions musculaires après avoir mangé des cailles, étant donné que celles-ci se nourrissent d'hellébore. Et cette même chose, je le sais, est aussi arrivée à Athènes à certains qui avaient consommé des cailles en trop grande abondance ${ }^{24}$.

Nous reviendrons plus loin sur ce passage qui, s'il confirme le lien entre les cailles et l'hellébore, confirme un effet, au pire, laxatif pour les consommateurs directs ou indirects (comme dans le cas des enfants nourris au sein) de certains ingrédients, à l'exception de ceux dont le tempérament parvient à en triompher et qui, comme les cailles, se révèlent capables de les digérer comme n'importe quelle nourriture. Et même si Galien note également l'apparition de tensions musculaires (sur lesquelle nous reviendrons également), on reste loin des conséquences observées dans le cas de la consommation de poisons mortels.

La question se pose donc de savoir où l'auteur de l'Anonyme a bien pu trouver mention de ces cailles mangeuses de ciguë, alors même que tous les médecins, après Galien, continuent de mentionner des cailles mangeuses d'hellébore. Tel est le cas de l'auteur de la Thériaque à Pison (début du III ${ }^{\mathrm{e}}$ siècle) lorsqu'il note que : "Certains médicaments ne font au départ absolument pas partie de l'alimentation humaine. C'est le cas de la ciguë dont se nourrissent les étourneaux sans que sa faculté mortifère s'exerce sur eux, tandis que nous, comme tu ne l'ignores pas, elle nous tue. Et l'hellébore, qui est une nourriture pour les cailles, détruit affreusement les hommes ${ }^{25}$. " Le Ps.-Alexandre d'Aphrodise observe toujours la même répartition entre les cailles qui se nourrissent d'un hellébore délétère pour les hommes et les étourneaux qui préfèrent la ciguë ${ }^{26}$, tandis que le Ps.-Caesarius ( $\mathrm{VI}^{\mathrm{e}}$ siècle), loue la nature de n'avoir rien produit d'inutile, pas même " la ciguë que mangent les étourneaux et l'hellébore que mangent les cailles $»^{27}$, et que l'auteur des Géoponiques ( $\mathrm{X}^{\mathrm{e}}$

${ }^{23}$ Gal. Comm.Epid. VI 5,35 (XVIIb 306,13-15 K. = CMG V.10.2.2, p. 317 Wenkebach/Pfaff) :

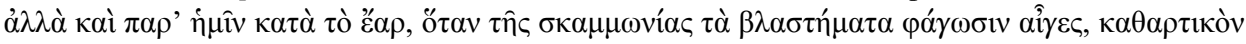

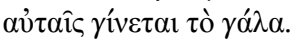

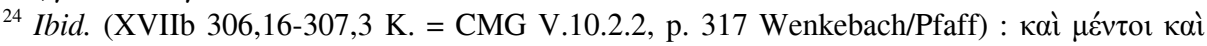

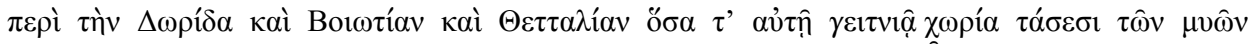

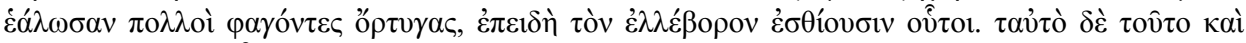

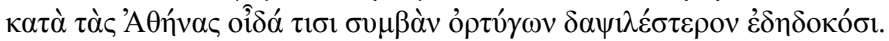

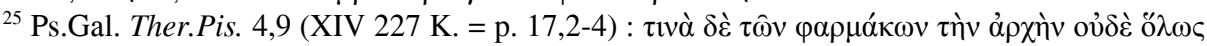

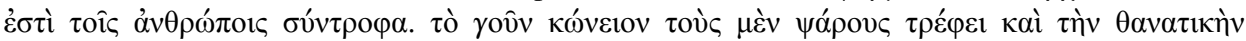

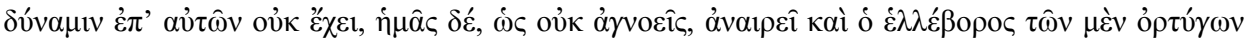

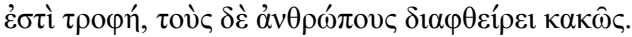

${ }^{26}$ Ps.Alex.Aphr. Probl. I, Prol. (Physici et medici Graeci minores I, p. 4,26 Ideler) : ő

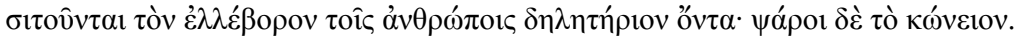

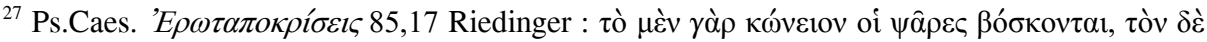
$\dot{\varepsilon} \lambda \lambda \varepsilon \dot{\beta} \beta$ ороv oi ő 
siècle), dans un passage peut-être inspiré par Didyme, mentionne encore « les cailles repues d'hellébore ${ }^{28}$.

\section{Quand la ciguë engraisse les cailles.}

Si ce n'est chez les médecins, où l'auteur de l'Anonyme a-t-il donc pu trouver des cailles mangeant de la ciguë ? En réalité en l'état de nos sources, et le fait vaut d'être souligné, le témoignage de l'Anonyme constitue la première attestation de «cailles mangeuses de ciguë », une attestation qui reste unique dans le reste de la littérature médicale où les cailles, on l'a vu, sont des mangeuses d'hellébore, mais qui trouve un écho chez le philosophe Sextus Empiricus (actif c. 190) qui était aussi médecin.

Chez Sextus, la mention des cailles intervient dans les Esquisses pyrrhoniennes, sorte de résumé de la doctrine sceptique, à l'intérieur du développement consacré aux «dix tropes dits d'Enésidème », dix modes ou schémas d'argumentation propres à conduire à la suspension du jugement. Les réactions différentes des différents êtres vivants dans divers domaines servent en effet à illustrer le fait que ceux-ci reçoivent des impressions différentes des mêmes objets, ce qui doit amener le philosophe à la «suspension (epokhê) de l'assentiment». De ce point de vue, les goûts différents des êtres vivants illustrent le fait que «d'une manière générale, ce qui est agréable à certains est pour d'autres désagréable, à éviter et même mortel ». Ainsi, poursuit Sextus, « la ciguë engraisse les cailles et la jusquiame ${ }^{29}$ les porcs ${ }^{30}$. Suivent les noms de plusieurs autres animaux dont les préférences alimentaires peuvent étonner (cerfs se nourrissant d'animaux venimeux, hirondelles de cantharides, ours de fourmis et de petits vers), à l'exclusion toutefois des étourneaux qui ne sont pas mentionnés dans cette énumération. Diogène Laërce (début III $^{\mathrm{e}}$ siècle) lui-même, dans la présentation qu'il donne de la philosophie sceptique et des fameux tropes, reprendra l'exemple des cailles mangeuses de ciguë, auxquelles il ajoutera le nom de deux seuls autres animaux : « La ciguë est nourrissante pour la caille, elle est mortelle pour l'homme. Le fumier est comestible pour le porc, non pour le cheval ${ }^{31}$.

Les deux témoignages parallèles de Sextus et Diogène Laërce, vraisemblablement puisés à une source commune, confirment donc la disparition des étourneaux au profit de la caille devenue mangeuse de ciguë. L'omission des étourneaux, traditionnellement mangeurs de ciguë, au moment même où cette plante est citée comme nourriture des cailles, n'est sans doute pas un hasard.

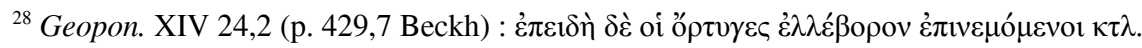

${ }^{29}$ Sur la jusquiame (hyoscyamos), littéralement « fève à porc », voir supra n. 9.

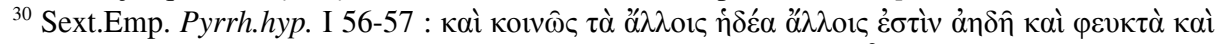

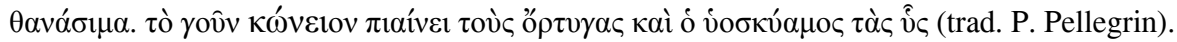

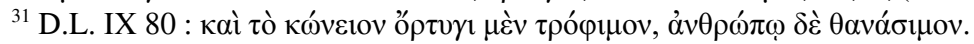


Une omission et/ou une erreur, déjà présente dans la source de Sextus et de Diogène, sont vraisemblablement à l'origine de cette nouvelle tradition des cailles mangeuses de ciguë dont, jusqu'alors, l'Anonyme était le seul représentant. L'auteur de l'Anonyme a-t-il puisé son renseignement sur les cailles à une source sceptique ? Si la question mérite d'être posée, il reste que le contexte de la citation, chez l'Anonyme et chez les philosophes sceptiques, est sensiblement différent puisqu'il n'est nulle part fait mention, dans les deux passages de Sextus et de Diogène, de la consommation par l'homme de cailles mangeuses de ciguë.

\section{La ciguë peut-elle être une nourriture?}

Les Anciens se sont pourtant très tôt demandés si les animaux qui se nourrissent de substances toxiques pouvaient être comestibles pour l'homme. Or, l'Anonyme n'est pas seulement le premier à mentionner des cailles mangeuses de ciguë, il est également le premier auteur médical grec à envisager la consommation de ces volatiles par les hommes ${ }^{32}$. A Rome, à la même époque, Pline (mort en 79) atteste aussi cette pratique, mais pour constater son abandon, apparemment pour des raisons sanitaires : «Les cailles sont très friandes d'une graine vénéneuse; c'est ce qui les a fait bannir des tables $»^{33}$.

Cependant, Pline ne précise ni la nature de la graine en question (hellébore ou ciguë), ni la nature des risques encourus ${ }^{34}$. Au siècle suivant, Galien est le premier médecin à mentionner les effets dangereux pour l'homme d'une consommation de cailles. On a en effet déjà vu plus haut que, dans son Commentaire aux Epidémies VI, le médecin de Pergame avait mis en relation une consommation importante de ces volatiles nourris d'hellébore avec

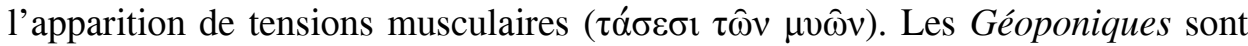
encore plus précis :

Et puisque les cailles qui sont repues d'hellébore mettent en danger ceux qui en mangent en tendant fortement leurs membres et en leur donnant des vertiges, il faut à chaque fois faire cuire avec elles du millet. Et si quelqu'un, pour en avoir mangé, a été préalablement saisi par cette affection, qu'il boive du millet en décoction ${ }^{35}$.

${ }^{32} \mathrm{Si}$ l'on excepte le passage de l'Ancien testament, tiré du livre de l'Exode où les Hébreux en fuite hors d'Egypte abusent des cailles envoyées par Yahvé pour les nourrir et meurent de s'être laissés aller à leur fringale.

${ }^{33}$ Plin. N.H. X 69 : Coturnicibus ueneni semen gratissimus cibus, quam ob causam eas damnauere mensae.

${ }^{34}$ Pline, ibid. se contente d'ajouter : «Une autre raison de cette proscription est l'épilepsie, à laquelle elles sont seules sujettes parmi les animaux, en dehors de l'homme. »

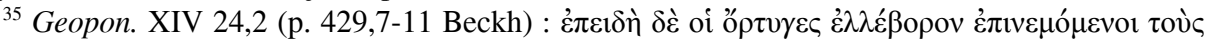


Les conséquences de cette intoxication alimentaire désignée sous le nom de coturnisme (de coturnix, la caille en latin) sont aujourd'hui bien connues. Elles se manifestent, entre autres, par une rhabdomyolyse, «c'est-à-dire une destruction massive et aiguë du tissu musculaire, voire des paralysies, se caractérisant par une faiblesse généralisée, de la fièvre, une extinction de voix, des douleurs musculaires, une paralysie des membres inférieurs, des vomissements, une décoloration des urines et/ou une gastro-entérite sévère $»^{36}$. En 1941, en Algérie, E. Sergent remarque que certains colons avaient développé divers troubles alimentaires, mais cependant jamais mortels, après avoir mangé des cailles. Il recourt à diverses expériences pour vérifier l'hypothèse couramment admise que les volatiles en question se seraient nourris de graines

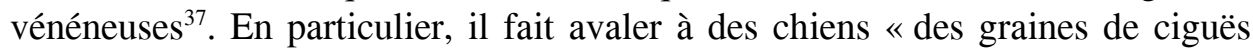
broyées dans un mortier dans 4 à 8 fois leur volume d'eau » (p. 187) et constate leur décès entre une heure et demie et huit heures et demie après l'ingestion. A l'opposé, il montre que les cailles peuvent absorber des quantités considérables de graines de ciguës sans éprouver le moindre malaise. En mai 1940, il fait manger à deux chiens des cailles qui avaient été gavées de ces graines vénéneuses (Conium maculatum). Les deux chiens, deux à quatre heures après, présentent des troubles évidents (ils ne pouvaient plus se maintenir sur leurs pattes postérieures), mais récupèrent après deux nouvelles heures. Quant aux graines incriminées dans ce genre d'intoxications, et comme le note E. Sergent qui a cependant fait ses expériences uniquement sur la ciguë, il s'agit de toute sorte de graines vénéneuses.

Toutefois si les expériences menées par E. Sergent dans les années quarante permettent d'éclairer le texte galénique, elles ne sont d'aucune utilité pour comprendre correctement le texte de l'Anonyme. Son auteur en effet, à la différence des autres médecins, n'envisage pas les effets toxiques d'une consommation de cailles nourries de ciguë, mais tire précisément argument de leur innocuité pour démontrer l'absurdité du raisonnement suivant : les cailles mangent de la ciguë, les hommes mangent les cailles, donc la ciguë est comestible. Ce syllogisme, on l'a vu, ne sert en réalité à l'auteur qu'à signaler une exception au principe selon lequel «tout se transforme en tout et est susceptible de devenir nourriture $\aleph^{38}$. Or cette façon de tirer argument de l'innocuité des cailles pour réfuter le fait que les drogues mortelles puissent être une nourriture constitue une seconde originalité de l'auteur de l'Anonyme qui là encore mérite d'être soulignée.

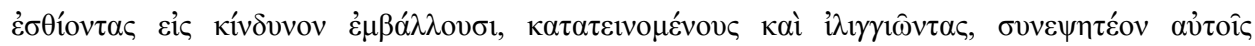

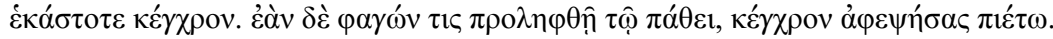

${ }^{36}$ Freney/Doléans-Jordheim, Cailles, 57.

${ }^{37}$ Sergent, Bible et France.

${ }^{38}$ Anon.Lond. xxvi 15-16. 
De fait, lorsque nos textes mentionnent la ciguë, c'est pour insister sur ses effets mortels sur l'homme, non pour l'envisager en tant que nourriture humaine, pas même de façon indirecte, par la médiation d'un animal consommateur. En réalité, la littérature médicale mentionne une seule exception, un seul cas de consommation (humaine) non mortelle de cette plante vénéneuse. Il est notamment rapporté par Galien qui précise qu'il était bien connu de tous. $\mathrm{Ce}$ récit intervient à l'intérieur des Facultés des médicaments simples dans le chapitre où Galien tente d'expliquer pourquoi la ciguë est mortelle pour l'homme. La ciguë agit par refroidissement et quand le froid gagne le cœur, il provoque son arrêt et la mort. Or ce processus ne s'observe pas chez tous les êtres vivants. Chez l'homme, du fait de la largeur des passages, de l'intensité de la chaleur vitale et de la robustesse des pulsations des artères, la drogue mortelle parvient au cœur rapidement et en quantité. À l'inverse, chez les étourneaux

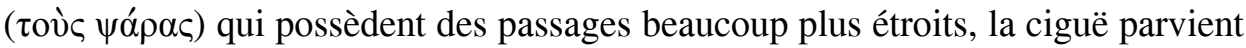
beaucoup plus lentement et en bien moindre quantité jusqu'au cœur qu'elle est ainsi incapable de refroidir dans des proportions suffisantes pour entraîner la mort. Tout est donc affaire de dose et, si la ciguë est prise en petite quantité, elle ne causera pas la mort d'un homme, ajoute Galien. Puis il rapporte le cas de cette vieille Athénienne qui prit d'abord régulièrement une toute petite quantité de ciguë, avant d'augmenter insensiblement les doses et de parvenir ainsi sans dommage à accoutumer son corps à la substance toxique ${ }^{39}$.

Le cas de cette vieille Athénienne paraît avoir fortement frappé les esprits et être devenu relativement célèbre. Sextus Empiricus lui-même s'en est fait l'écho :

Il y avait, dit-on, en Attique une vieille femme qui consommait le poids de trente drachmes de ciguë sans danger, et Lysis prenait celui de quatre drachmes de pavot sans dommage ${ }^{40}$.

L'anecdote n'est pas non plus restée ignorée des Byzantins, en particulier de Michel Psellos qui la mentionne deux fois et de Michel Choniatès ${ }^{41}$. Mais le

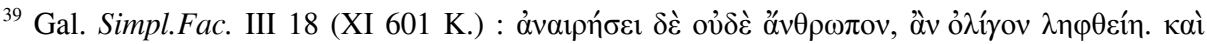

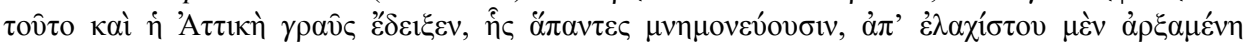

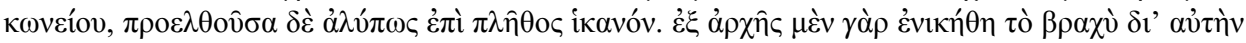

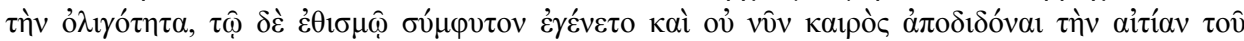

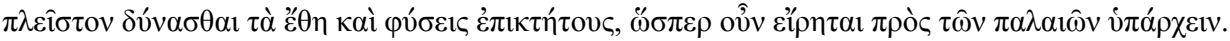

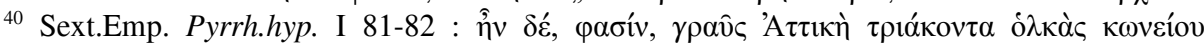

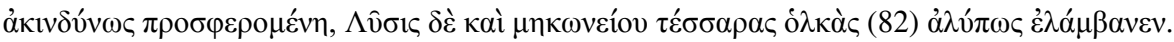

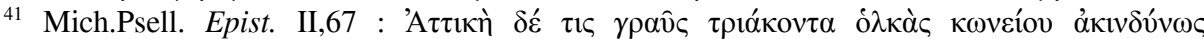

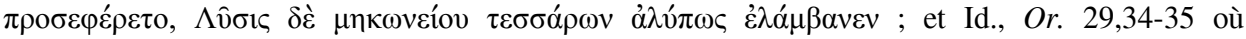

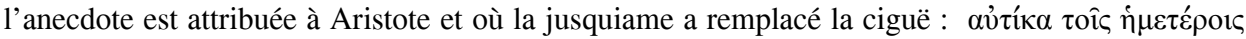

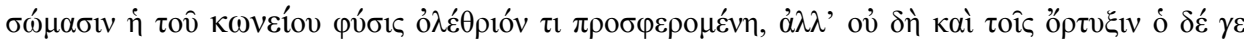

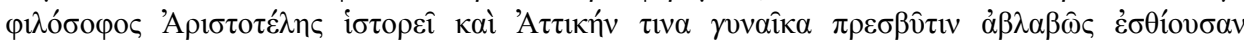


contexte dans lequel est rapportée cette expérience, que la science moderne désigne sous le nom de mithridatisation, apparaît différent de celui de l'Anonyme et des mécanismes de la nutrition. D'ailleurs, le récit de la vieille Athénienne n'envisage qu'une consommation directe de ciguë, sans référence aux cailles qui, dans ces récits, ont ou bien purement et simplement disparu ou, comme chez Galien, été remplacées par les étourneaux.

En conclusion, l'auteur de l'Anonyme ne tire pas argument des cailles mangeuses de ciguë pour démontrer le caractère toxique on non de ces volatiles et encore moins pour illustrer un cas remarquable de mithridatisation. Cet exemple n'a d'autre but, dans le contexte de l'Anonyme, que de démontrer que les drogues mortelles, au même titre que le bois et la pierre, ne peuvent jamais être de la nourriture pour l'homme, pas même prises en très petites quantités (précision que l'auteur de l'Anonyme ne donne pas, car il n'a pas songé à l'envisager). À l'intérieur de cet exposé consacré aux mécanismes de la nutrition, l'auteur de l'Anonyme, en l'état de nos sources, apparaît donc comme le premier à mentionner des cailles mangeuses de ciguë, quand les auteurs médicaux citent traditionnellement les étourneaux. Mais surtout, pour démontrer l'absurdité du principe selon lequel « tout se transforme en tout», il n'hésite pas à sortir de son contexte, à détourner et à adapter un exemple traditionnel de la littérature médicale (les cailles mangeuses d'hellébore et les étourneaux mangeurs de ciguë) pour illustrer l'impossibilité logique qu'il y aurait à ériger la ciguë (tout comme le bois et la pierre) au rang de nourriture humaine. Cette capacité de l'auteur de l'Anonyme à se détacher de ses sources et à recourir à un exemple traditionnel de la littérature pharmacologique pour illustrer un raisonnement personnel et original, dont on ne trouve la trace nulle part ailleurs, méritait d'être soulignée.

\section{Bibliographie}

André, Plantes = Jacques André, Les noms de plantes dans la Rome antique, Paris 1985.

Drossaart/Poortman, De plantis = Nicolas de Damas, De plantis: Five Translations, ed. and introd. by H. J. Drossaart and E. L. J. Poortman, Amsterdam/Oxford 1989.

Freney/Doléans-Jordheim, Cailles = Jean Freney / Anne Doléans-Jordheim, «Les cailles empoisonneuses, le vin au plomb ou le miel qui rend fou! Histoire de quelques intoxications alimentaires inexpliquées de l’Antiquité, « Revue de Biologie Médicale », 316 (Janvier 2014), 55-60.

Kotsia-Pantele, P.Lond. 137 = P. Kotsia-Pantele, P.Lond. 137 (= P.Lit.Lond. 165) XXVI 16-19, «Hellenika » 40 (1989), 149-53 (article) et 222 (résumé).

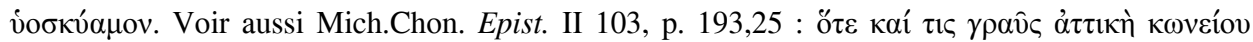

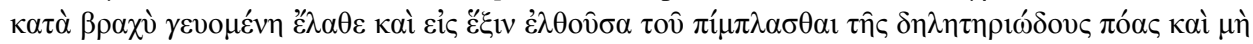
$\dot{\alpha} \pi \hat{\lambda} \lambda \lambda v \sigma \theta \alpha 1$. 
Manetti, Asclepiade = Daniela Manetti, Il ruolo di Asclepiade di Bitinia nell'Anonimo Londinese, in : A. Garzya / J. Jouanna (édd.), Transmission et ecdotique des textes médicaux grecs, Napoli 2003, 335-47.

Manetti, Autografi = Daniela Manetti, Autografi e incompiuti: il caso dell'Anonimo Londinese $\mathrm{P}$. Lit. Lond. 165, ZPE 100 (1994), 47-58.

Manetti, Anonymus = Daniela Manetti (éd.), Anonymus Londiniensis, Berlin/New York 2011.

Marganne, Acquisition = Marie-Hélène Marganne, Les modes d'acquisition et de transmission du savoir médical dans l'antiquité gréco-romaine, «Histoire des sciences médicales » 51.2 (2017), 163-73.

Nissen, Asclépios = Cécile Nissen, Entre Asclépios et Hippocrate. Étude des cultes guérisseurs et des médecins de Carie, Liège 2009.

Ricciardetto, Anonyme = Antonio Ricciardetto, L'Anonyme de Londres. P.Lit.Lond. 165, Brit . Libr. inv. 137. Un papyrus médical grec du $I^{e r}$ siècle après J.-C., Paris 2016.

Sergent, Bible $=$ Edmond Sergent, Les cailles empoisonneuses dans la Bible, et en Algérie de nos jours. Aperçu historique et recherches expérimentales, "Archives de l'Institut Pasteur d'Algérie » 19 (1941), 161-92.

Sergent, France $=$ Edmond Sergent, Les Cailles empoisonneuses en France : deuxième note, « Archives de l'Institut Pasteur d'Algérie » 26 (1948), 249-52.

Thompson, Glossary = D'Arcy Wentworth Thompson, A Glossary of Greek Birds, Oxford 1895. 